\title{
Special normal form of a hyperbolic CR-manifold in $\mathbb{C}^{4}$
}

\author{
by Vladimir V. Ežov (Adelaide, S.A.) and Gerd Schmalz (Bonn)
}

\begin{abstract}
We give a special normal form for a non-semiquadratic hyperbolic CRmanifold $M$ of codimension 2 in $\mathbb{C}^{4}$, i.e., a construction of coordinates where the equation of $M$ satisfies certain conditions. The coordinates are determined up to a linear coordinate change.
\end{abstract}

Introduction. In 1907 Poincaré [Poi07] posed the problem of local biholomorphic equivalence of two real-analytic hypersurfaces in $\mathbb{C}^{2}$. It is interesting to consider this problem for real hypersurfaces in $\mathbb{C}^{N}$ or, more generally, for real-analytic CR-submanifolds in $\mathbb{C}^{N}$. One approach to this problem, due to Chern and Moser [CM74], is the construction of normal forms of the hypersurfaces, i.e., special coordinate systems with the property that certain monomials in the equation of the hypersurface, considered as a power series, vanish. This construction is universal for all hypersurfaces with a given signature of the Levi form. The normal form condition determines the coordinate system up to a rather complicated action of the finite-dimensional isotropy group of the quadric of maximal osculation. This group is covered by $\mathrm{SU}(n-p, p+1)$, where $n=N-1$ and $p$ is the signature of the Levi form. If both hypersurfaces are given in normal form the equivalence problem reduces to the (still complicated) problem of finding a transformation out of a finite-dimensional variety that maps one hypersurface to the other.

Webster [Web78] introduced in 1978 a special normal form that takes into account higher order terms of a given hypersurface. By imposing additional requirements he reduces the freedom in the choice of normal coordinates to a subgroup of $\mathrm{GL}(n, \mathbb{C})$ that acts by linear transformations.

1991 Mathematics Subject Classification: Primary 32C16; Secondary 32C05, 32H02.

Key words and phrases: CR-manifolds, invariants, classification.

Research of the first author was supported by ARC and SFB 256.

Research of the second author was supported by SFB 256 of Deutsche Forschungsgemeinschaft. 
In this paper we construct a special normal form for hyperbolic CR-manifolds of codimension 2 in $\mathbb{C}^{4}$. By a hyperbolic $C R$-manifold we mean a CR-submanifold $M \subset \mathbb{C}^{4}$ that can be written in the form

$$
v_{1}=\left|z_{1}\right|^{2}+N_{1}(z, \bar{z}, u), \quad v_{2}=\left|z_{2}\right|^{2}+N_{2}(z, \bar{z}, u),
$$

where $z_{1}, z_{2}, w_{1}, w_{2}$ are coordinates in $\mathbb{C}^{4}, w_{j}=u_{j}+i v_{j}$ and $N_{j}$ does not contain any terms of order less than 3 . Hyperbolic CR-manifolds in $\mathbb{C}^{4}$ can also be characterised as the strictly pseudoconvex CR-manifolds of codimension 2 in $\mathbb{C}^{4}$. For such manifolds Loboda [Lob88] constructed a normal form that is analogous to Chern-Moser's normal form for hypersurfaces. Loboda's normal form conditions can be described as follows:

Set

$$
N_{j}(z, \bar{z}, u)=N_{j}^{1}\left(z_{j}, \bar{z}_{j}, u_{j}\right)+N_{j}^{2}(z, \bar{z}, u),
$$

where $N_{j}^{1}\left(z_{j}, \bar{z}_{j}, u_{j}\right)$ consists of all monomials that depend only on $z_{j}, \bar{z}_{j}, u_{j}$. Then

$$
N_{j}^{1}=2 \operatorname{Re}\left(h_{42}^{j}(u) z_{j}^{4} \bar{z}_{j}^{2}\right)+\sum_{\substack{k, l \geq 2 \\ k+l \geq 7}} h_{k l}^{j}(u) z_{j}^{k} \bar{z}_{j}^{l},
$$

where $h_{k l}^{j}(u)$ are real-analytic functions of $u$. Notice that these are exactly Chern-Moser's conditions for the normal form of a hypersurface in $\mathbb{C}^{2}$.

For the terms $N_{j}^{2}$ the following holds:

$$
\begin{aligned}
\left(N_{j}^{2}\right)_{k 0} & \equiv 0 \quad \text { for } k \geq 1, & \left(N_{j}^{2}\right)_{11} \equiv 0, \\
\frac{\partial}{\partial \bar{z}_{j}}\left(N_{j}^{2}\right)_{k 1} & \equiv 0 \quad \text { for } k \geq 2, & \frac{\partial^{2}}{\partial z_{1} \partial z_{2}}\left(N_{j}^{2}\right)_{21} \equiv 0, \\
\frac{\partial^{4}}{\partial z_{1} \partial z_{2} \partial \bar{z}_{1} \partial \bar{z}_{2}}\left(N_{j}^{2}\right)_{22} & \equiv 0, &
\end{aligned}
$$

We denote by $\mathcal{N}_{0}(M)$ the family of biholomorphic, origin preserving transformations that map $M$ (in normal form) into $M^{\prime}$ that also has normal form. If $\Phi \in \mathcal{N}_{0}(M)$ is given by $z^{\prime}=f(z, w), w^{\prime}=g(z, w)$ then

$$
L_{C}: \quad z^{\prime}=C z, \quad w^{\prime}=\varrho w
$$

with

$$
C=\left.\left(\frac{\partial f}{\partial z}\right)\right|_{0}, \quad \varrho=\left.\left(\frac{\partial g}{\partial w}\right)\right|_{0}
$$

also belongs to $\mathcal{N}_{0}(M)$. Moreover, $C$ and $\varrho$ must then have the form

or

$$
C=\left(\begin{array}{cc}
\lambda_{1} e^{i \psi_{1}} & 0 \\
0 & \lambda_{2} e^{i \psi_{2}}
\end{array}\right), \quad \varrho=\left(\begin{array}{cc}
\lambda_{1}^{2} & 0 \\
0 & \lambda_{2}^{2}
\end{array}\right),
$$

$$
C=\left(\begin{array}{cc}
0 & \lambda_{1} e^{i \psi_{1}} \\
\lambda_{2} e^{i \psi_{2}} & 0
\end{array}\right), \quad \varrho=\left(\begin{array}{cc}
0 & \lambda_{1}^{2} \\
\lambda_{2}^{2} & 0
\end{array}\right)
$$


where $\lambda_{j}>0, \psi_{j} \in \mathbb{R}$. The group of all $(C, \varrho)$-transformations will be denoted by $\operatorname{GL}(Q)$.

Thus, any $\Psi \in \mathcal{N}_{0}(M)$ can be decomposed into $\Psi=L_{C} \circ \Psi_{\text {id }}$ with $\Psi_{\text {id }} \in \mathcal{N}_{0, \text { id }}(M)$, where

$$
\mathcal{N}_{0, \text { id }}(M)=\left\{\Phi \in \mathcal{N}_{0}(M):\left.\left(\frac{\partial f}{\partial z}\right)\right|_{0}=\mathrm{id}\right\} .
$$

It follows from Loboda's theorem that any $\Phi \in \mathcal{N}_{0 \text {,id }}(M)$ is uniquely determined by the data

$$
a_{j}:=\left.\frac{\partial f_{j}}{\partial w_{j}}\right|_{0}, \quad r_{j}:=\left.\frac{1}{2} \frac{\partial^{2} g_{j}}{\left(\partial w_{j}\right)^{2}}\right|_{0} .
$$

If $M$ is the hyperbolic quadric

$$
M: \quad v_{1}=\left|z_{1}\right|^{2}, \quad v_{2}=\left|z_{2}\right|^{2}
$$

then for any possible initial data there exists an automorphism, hence any normalisation is automatically an automorphism.

For arbitrary $M$ the right hand side of equation (1) can be expanded into a series of homogeneous polynomials where the weighted degree of each monomial is the sum of the degrees with respect to $z$ and $\bar{z}$ and twice the degree with respect to $u$. If $M$ is non-quadratic and given in normal form then there is a smallest weight $\gamma$ such that the homogeneous component $N_{\gamma}$ with weight $\gamma$ of $N=\left(N^{1}, N^{2}\right)$ is different from 0 . We will refer to this $N_{\gamma}$ as to the first non-quadratic term. It can be easily verified that any normalisation $\Phi \in \mathcal{N}_{0}(M)$ is equal to the quadratic automorphism with the same initial data up to terms of weighted degree $\geq \gamma$ (for more elaborate explanation see [Sch95]).

Among the non-quadratic hyperbolic manifolds there is a special type of manifolds that are called semiquadratic manifolds and should be separately dealt with. A semiquadratic manifold is given by the equations

$$
v_{1}=\left|z_{1}\right|^{2}, \quad v_{2}=\left|z_{2}\right|^{2}+N^{2}\left(z_{2}, \bar{z}_{2}, u_{2}\right),
$$

where $z_{1}, z_{2}, w_{1}, w_{2}$ are normal coordinates, i.e., they are a Cartesian product of a sphere in $\mathbb{C}^{2}$ and a non-spherical hypersurface in $\mathbb{C}^{2}$ that is given in Chern-Moser's normal form.

The construction of a special normal form reduces then to Chern-MoserWebster's special normal form for hypersurfaces. A coordinate change from one special normal form to another is a fractional linear sphere automorphism in the variables $z_{1}, w_{1}$ and a linear transformation in the variables $z_{2}, w_{2}$.

Now, we may restrict ourselves to the case of non-semiquadratic manifolds. The construction and the condition of the special normal form for a given non-semiquadratic manifold $M$ depend on the concrete form of the 
first non-quadratic, resp. non-semiquadratic, term. We will consider 4 different cases of non-semiquadratic manifolds that appeared in [Sch95] where it was proved that for such manifolds there exist normal coordinates where all automorphisms are linear. This is, of course, necessary for a construction of special normal forms with the property that passing to another special normal coordinates is provided by linear transformations because any automorphism is in particular a transformation to other special normal coordinates.

In Section 2 we recall the rough scheme of the proof of the following theorem from [Sch95]:

THEOREM 1. Let $M$ be a non-semiquadratic, real-analytic $C R$-manifold in $\mathbb{C}^{4}$. Then any isotropic automorphism $\Phi=(f, g)$, considered as a normalisation, is uniquely determined by the parameter

$$
C=\left.\frac{\partial f}{\partial z}\right|_{0} .
$$

Section 3 is devoted to the description of the special normal form and to the proof of the existence and uniqueness (up to linear coordinate changes) of special coordinates.

We remark that a special normal form for non-umbilic hyperbolic CR-manifolds of codimension 2 in $\mathbb{C}^{4}$ was constructed by Loboda in [Lob90].

2. Sketch of the proof of Theorem 1. The proof of Theorem 1 is very long and very technical. Here we only sketch the main ideas.

STEP 1. One has to show that any automorphism with $C=$ id is trivial. Any such automorphism can be embedded in some one-parameter subgroup $\Phi_{t}$. From

(3) $\operatorname{Im} g_{t}(z, w)-\left\langle f_{t}(z, w), f_{t}(z, w)\right\rangle-\left.N\left(f_{t}, \bar{f}_{t}, \operatorname{Re} g_{t}\right)\right|_{\operatorname{Im} w=\langle z, z\rangle+N} \equiv 0$

one deduces that the derivative with respect to $t$ must vanish for $t=0$. Denote this function by $P(z, \bar{z}, u)$.

STEP 2. The function $P(z, \bar{z}, u)$ can be expressed as

$$
P(z, \bar{z}, u)=\left.2 \operatorname{Re} \chi(v-\langle z, z\rangle-N(z, \bar{z}, u))\right|_{\operatorname{Im} w=\langle z, z\rangle+N},
$$

where $\chi$ is a vector field that decomposes into a series of homogeneous components with respect to the weight

$$
\chi=\chi_{1}+\chi_{2}+\chi_{\gamma-1}+\ldots
$$

Here, one has to take into account that the basis vectors $\partial / \partial z_{j}$, resp. $\partial / \partial w_{j}$, have weight -1 , resp. -2 . Then the index indicates the weight of the compo- 
nent. The vector fields $\chi_{1}$ and $\chi_{2}$ do not depend on $M$ and have the explicit form

$$
\begin{aligned}
\chi_{1} & =\sum_{j=1,2}\left(a_{j} w_{j}+2 i \bar{a}_{j}\left(z_{j}\right)^{2}\right) \frac{\partial}{\partial z_{j}}+2 i \bar{a}_{j} z_{j} w_{j} \frac{\partial}{\partial w_{j}}, \\
\chi_{2} & =\sum_{j=1,2} r_{j} z_{j} w_{j} \frac{\partial}{\partial z_{j}}+r_{j}\left(w_{j}\right)^{2} \frac{\partial}{\partial w_{j}} .
\end{aligned}
$$

STEP 3. Besides the weighted degree, there is another characteristic of a monomial that plays an important rôle in the proof. It is called the $j$-defect (for $j=1,2$ ) and is defined as the difference of the degrees in $z_{j}$ and in $\bar{z}_{j}$.

Now, suppose that $a_{j} \neq 0$. Without loss of generality one can assume that $a_{1}=i$. One has to show that then equation (3) cannot be satisfied. This implies that there are no isotropic automorphisms $\Phi$ of $M$ with $\Phi \in$ Aut $_{0, \text { id }}$. We distinguish four different cases:

1. $M$ has the form

$$
v_{1}=\left|z_{1}\right|^{2}+N^{1}(z, \bar{z}, u), \quad v_{2}=\left|z_{2}\right|^{2}+N^{2}(z, \bar{z}, u),
$$

where $N_{\gamma_{1}, \delta_{1}}^{1} \not \equiv 0$ is the term of maximal 1-defect $\delta_{1}+1$ of the first nonvanishing term of $N^{1}$. Then the term in $P^{1}$ of weight $\gamma_{1}$ and 1-defect $\delta_{1}$ takes the form

$P_{\gamma_{1}+1, \delta_{1}+1}^{1}=\left.\chi_{(1)}\right|_{v_{1}=\left|z_{1}\right|^{2}} N_{\gamma_{1}, \delta_{1}}^{1}-2 z_{1} N_{\gamma_{1}, \delta_{1}}^{1}+\left.\chi_{\gamma_{1}-1, \delta_{1}+1}\right|_{v_{1}=\left|z_{1}\right|^{2}}\left(\left|z_{1}\right|^{2}-v_{1}\right)$, where

$$
\chi_{(j)}=i \bar{a}_{j}\left(2\left(z_{j}\right)^{2} \frac{\partial}{\partial z_{j}}+2\left(u_{j}+i v_{j}\right) z_{j} \frac{\partial}{\partial w_{j}}-i\left(u_{j}-i v_{j}\right) \frac{\partial}{\partial \bar{z}_{j}}\right)
$$

is the part of the vector field $\chi_{1}$ that increases the $j$-defect by 1 .

Due to (3), this expression must vanish. For the first case we suppose that the sum of the terms including $N^{1}$ is different from 0 . Then they must be compensated by the last term that includes $\chi_{\gamma_{1}-1, \delta_{1}+1}$. This turns out to be impossible because then the last term has non-normal components that cannot occur in the first two terms. Hence, $a_{1} \neq 0$ implies that the polynomials $P_{\gamma_{1}+1, \delta_{1}+1}^{1}$ cannot vanish, i.e., for $a_{1}$ running over $\mathbb{C}$ they form a one-dimensional subspace in the space of polynomials with weight $\gamma_{1}+1$ and $j$-defect $\delta_{1}+1$.

2. For the second case we assume that $M$ has the form

$$
v_{1}=\left|z_{1}\right|^{2}, \quad v_{2}=\left|z_{2}\right|^{2}+N^{2}(z, \bar{z}, u),
$$

where $N_{\gamma_{2}, \delta_{2}}^{2} \not \equiv 0$ is the first non-vanishing term with maximal 2-defect, $N_{\gamma_{1}, \delta_{1}}^{2}$ is the first term that depends on one of the variables $z_{1}, \bar{z}_{1}, u_{1}$ with weight $\gamma_{1}$ and maximal 1-defect $\delta_{1}$. Then $a_{1} \neq 0$ implies $P_{\gamma_{1}+1, \delta_{1}+1}^{2} \neq 0$ (even without the assumption $N^{1} \equiv 0$ ). Furthermore, the second case can 
be characterised by $a_{2} \neq 0$ implies $P_{\gamma_{2}+1, \delta_{2}+1}^{2} \neq 0$, as above. In this case the polynomials $P_{\gamma_{j}+1, \delta_{j}+1}^{j}$ form one-dimensional subspaces in the spaces of polynomials with weight $\gamma_{j}+1$ and $j$-defect $\delta_{j}+1$ for $a_{j}$ running over $\mathbb{C}$.

3. For the third case $M$ has the form (4) but $a_{1} \neq 0$ does not imply that $P_{\gamma_{1}+1}^{1} \neq 0$. Then one finds that $N_{\gamma_{1}}^{1}$ must have the special form

$$
v_{1}=\left|z_{1}\right|^{2}+\left(u_{1}+i\left|z_{1}\right|^{2}\right)\left(u_{1}-i\left|z_{1}\right|^{2}\right) \Pi_{2}\left(z_{2}, \bar{z}_{2}, u_{2}\right)+\ldots,
$$

where $\Pi_{2}$ is not constant and depends only on the indicated variables. Then it follows from $a_{2} \neq 0$ that $P_{\gamma_{1}+1}^{1} \neq 0$. There are two possibilities for $N^{2}$ : either there is a first component $N_{\gamma_{2}}^{2}$ that depends on one of the variables $z_{1}, \bar{z}_{1}, u_{1}$ and $a_{1} \neq 0$ implies $P_{\gamma_{2}+1}^{2} \neq 0$, or $N^{2}$ depends only on $z_{2}, \bar{z}_{2}, u_{2}$. The first alternative characterises the third case. Then one finds out that $a_{j}$ running over $\mathbb{C}$ produces one-dimensional subspaces of $P_{\gamma_{j}+1, \delta_{j}+1}^{l}(j \neq l)$ in the space of polynomials with weight $\gamma_{j}+1$ and $j$-defect $\delta_{j}+1$.

4. The second alternative above constitutes the fourth case. Then there exists a number $k$ with $2 \leq k \leq \gamma$ such that $P_{\gamma_{1}+k}^{1} \neq 0$. We will call $M$ exceptional of order $k$.

STEP 4. If $r_{j} \neq 0$ then $P_{\gamma+2} \neq 0$.

3. The special normal form. Consider the vector fields

$$
\begin{aligned}
\chi_{(j)} & =i \bar{a}_{j}\left(2\left(z_{j}\right)^{2} \frac{\partial}{\partial z_{j}}+2\left(u_{j}+i v_{j}\right) z_{j} \frac{\partial}{\partial w_{j}}-i\left(u_{j}-i v_{j}\right) \frac{\partial}{\partial \bar{z}_{j}}\right), \\
\chi_{2}^{j} & =r_{j} w_{j} z_{j} \frac{\partial}{\partial z_{j}}+r_{j}\left(w_{j}\right)^{2} \frac{\partial}{\partial w_{j}}, \\
\chi_{\gamma} & =f_{\gamma+1}(z, w) \frac{\partial}{\partial z}+g_{\gamma+2}(z, w) \frac{\partial}{\partial w}, \quad \gamma \geq 3,
\end{aligned}
$$

with $a_{j} \in \mathbb{C}, r_{j} \in \mathbb{R}$. Notice that $\chi_{(j)}$ increases the $j$-defect of a monomial by 1 and that

$$
2 \operatorname{Re} \chi_{1}=\chi_{(1)}+\chi_{(2)}+\bar{\chi}_{(1)}+\bar{\chi}_{(2)}, \quad \chi_{2}=\chi_{2}^{1}+\chi_{2}^{2} .
$$

We denote by $\chi_{\gamma, \delta_{j}}$, resp. $\chi_{\gamma, d}$, the component of the $j$-defect $\delta_{j}$, resp. of the total defect $d=\delta_{1}+\delta_{2}$, in $\chi_{\gamma}$.

We now distinguish four types of manifolds in dependence on the first non-quadratic terms in the expansion of the equation in normal form. Since the first order term of any renormalisation is a linear mapping $L_{C}$ as described above, the first non-quadratic terms in the equation of $M$ depend in a simple way on the normal coordinates chosen: only the coefficients of the monomials change. Therefore, the definitions below are correct.

These four types of manifolds are according to the four cases in Step 3 of the proof above. 
1. Both $N^{j} \not \equiv 0$ and do not have the special form indicated in case 3 . Let $N_{\gamma_{j}}^{j}$ be the first non-quadratic terms. Collect in $N_{\gamma_{j}}^{j}$ the terms of maximal total defect $d_{j}$, then within this polynomial the terms of maximal $j$-defect $\delta_{j}$ and then the terms of maximal weight $\omega_{j}$ with respect to $\left(z_{j}, \bar{z}_{j}, u_{j}\right)$. Finally, in the resulting expression choose a polynomial of the form

$$
\widehat{N}_{\gamma_{j}, d_{j}}^{j}=\widetilde{N}_{\omega_{j}, \delta_{j}}^{j}\left(z_{j}, \bar{z}_{j}, u_{j}\right) \Pi^{j}
$$

where $\Pi^{j}$ is a monomial in $z_{l}, \bar{z}_{l}, u_{l}(l \neq j)$ of minimal degree with respect to $z_{l}, \bar{z}_{l}$. Consider the space

$$
\text { (9) } \begin{aligned}
\mathcal{P}^{j}=\left\{P_{\gamma_{j}+1, \delta_{j}+1}^{j}=\chi_{(j)} \widehat{N}_{\gamma_{j}, \delta_{j}}^{j}\right. & -2 z_{j} \widehat{N}_{\gamma_{j}, \delta_{j}}^{j} \\
& \left.+\left.\chi_{\gamma_{j}-1, \delta_{j}+1}\left(\left|z_{j}\right|^{2}-v_{j}\right)\right|_{v_{j}=\left|z_{j}\right|^{2}}\right\},
\end{aligned}
$$

where $\chi_{\gamma_{j}-1, \delta_{j}+1}$ is uniquely determined for given $a_{j}$ by the condition that $P^{j}$ consists of normal terms. The arguments from [Sch95] (cf. Section 2) applied to normalisations (instead of automorphisms) show that these spaces are 1-dimensional complex spaces for $a_{j}$ running over $\mathbb{C}$. We can make use of these arguments because the actions of automorphisms and normalisations on the first non-quadratic terms are the same.

Analogously, we define the 1-dimensional real spaces

$$
\mathcal{Q}^{j}=\left\{Q_{\gamma_{j}+2, \delta_{j}}^{j}=\left.\chi_{2}^{j} \widehat{N}_{\gamma_{j}, d_{j}}^{j}\right|_{v_{j}=\left|z_{j}\right|^{2}}\right\} .
$$

2. One of the $N^{j}$ is 0 , say $N^{1} \equiv 0$. Since $M$ is not semiquadratic, $N^{2}$ must then depend on one of the $z_{1}, \bar{z}_{1}, u_{1}$. We define $\widehat{N}^{2}$ in the same way as above. $\widehat{N}^{1}$ will be defined analogously, but starting from $N_{\gamma_{1}, \delta_{1}}^{2}$, the term in $N^{2}$ of minimal weight $\gamma_{1}$ and maximal 1-defect $\delta_{1}$ that depends on one of the variables $z_{1}, \bar{z}_{1}, u_{1}$. Now, we define by (9), resp. (10), the 1-dimensional complex spaces $\mathcal{P}^{j}$, resp. the 1 -dimensional real spaces $\mathcal{Q}^{j}$.

3. One of the $N^{j}$ has the special form from case 3 and, therefore, must depend on one of the variables $z_{l}, \bar{z}_{l}, u_{l}(l \neq j)$, and $N^{l}$ depends on $z_{j}, \bar{z}_{j}, u_{j}$. $\widehat{N}^{j}$ is then derived from $N_{\gamma_{j}, \delta_{j}}^{l}(l \neq j)$, the component of minimal weight and maximal $j$-defect that contains one of the variables $z_{j}, \bar{z}_{j}, u_{j}$.

The spaces $\mathcal{P}^{j}$ and $\mathcal{Q}^{j}$ will also be defined by (9) and (10). According to $[\mathrm{Sch} 95], \mathcal{P}^{j}$ are complex 1 -dimensional for $a_{j}$ running over $\mathbb{C}$ and the $\mathcal{Q}^{j}$ are real 1-dimensional for $r_{j}$ running over $\mathbb{R}$.

4. One of the $N^{j}$, say $N^{1}$, has the special form from case 3 , and $N^{2}$ does not depend on $z_{1}, \bar{z}_{1}, u_{1}$. Then there exist an integer $k$ such that the spaces generated by $P_{\gamma_{1}+k, 1}^{1}$, resp. $P_{\gamma_{1}+1, \delta_{2}+1}^{1}$, for $a_{1}$, resp. $a_{2}$, in $\mathbb{C}$ are one-dimensional. In this case $\widehat{N}^{2}, \mathcal{P}^{2}$ and $\mathcal{Q}^{j}$ are defined as for manifolds of type 3. $\widehat{N}^{1}$ will be derived from $N_{\gamma_{1}, \delta_{1}}^{1}\left(\gamma_{1}=\gamma_{2}+k-1\right)$, the term of minimal weight and maximal 1-defect that produces a one-dimensional space of polynomials $P_{\gamma_{2}+k, 1}$. 
Set

$$
\begin{aligned}
\mathcal{P}^{1}=\left\{P_{\gamma_{1}+k, \delta_{1}+1}^{1}=\chi_{(j)} \widehat{N}_{\gamma_{j}, \delta_{j}}^{1}-2 z_{1}\right. & \widehat{N}_{\gamma_{1}+k-1, \delta_{1}}^{1} \\
& \left.+\left.\chi_{\gamma_{1}+k-2, \delta_{1}+1}\left(\left|z_{1}\right|^{2}-v_{1}\right)\right|_{v_{1}=\left|z_{1}\right|^{2}}\right\} .
\end{aligned}
$$

In the spaces of polynomials with given weight we introduce an inner product such that the monomials form an orthonormal basis. We will use this inner product in order to choose complementary subspaces.

Now we are ready to give the definition of the s.n.f.

Definition 1. A non-semiquadratic CR-manifold $M$ has special normal form (s.n.f.) if for some choice of $\widehat{N^{j}}$ the following conditions are satisfied:

- If $M$ is of type 1 , then

$$
N_{\gamma_{j}+1, \delta_{j}+1}^{j} \in \mathcal{P}^{j \perp} \quad \text { and } \quad N_{\gamma_{j}+2, \delta_{j}}^{j} \in \mathcal{Q}^{j \perp} .
$$

- If $M$ is of type 2 , then

$$
N_{\gamma_{j}+1, \delta_{j}+1}^{2} \in \mathcal{P}^{j \perp} \quad \text { and } \quad N_{\gamma_{j}+2, \delta_{j}}^{2} \in \mathcal{Q}^{j \perp} .
$$

- For $M$ of type 3 ,

$$
N_{\gamma_{j}+1, \delta_{j}+1}^{l} \in \mathcal{P}^{j \perp} \quad \text { and } \quad N_{\gamma_{j}+2, \delta_{j}}^{l} \in \mathcal{Q}^{j \perp} \quad(l \neq j) .
$$

- For $M$ of exceptional type,

$$
N_{\gamma_{1}+k, \delta_{1}+1}^{1} \in \mathcal{P}^{1 \perp}, \quad N_{\gamma_{1}+1, \delta_{2}+1}^{1} \in \mathcal{P}^{2 \perp}, \quad N_{\gamma_{j}+2, \delta_{j}}^{j} \in \mathcal{Q}^{j \perp} .
$$

By definition $M$ also has s.n.f. if its equation can be obtained from one of the equations above by interchanging $z_{1}$ with $z_{2}$ and $w_{1}$ with $w_{2}$.

THEOREM 2. If $M$ is a hyperbolic non-semiquadratic $C R$-manifold in $\mathbb{C}^{4}$ of codimension 2 then there exist coordinates such that the equation of $M$ has special normal form. The coordinates where $M$ has s.n.f. are determined up to a linear transformation of the form (2).

Proof. Let $M$ be given in normal form in the sense of Loboda and let $\Phi_{a}=\left(f_{a}, g_{a}\right)$ be a normalisation of $M$ with parameters $(C=\mathrm{id}, a=$ $\left.\left(a_{1}, a_{2}\right), r=0\right)$. The expansion of this normalisation into weighted homogeneous components differs from a quadratic automorphism only starting from terms of weight $\gamma$ in $f_{a}$ and of weight $\gamma+1$ in $g_{a}$. The spaces $\mathcal{P}^{j}$ were chosen so that for suitable $a_{1}, a_{2}$ the $\mathcal{P}^{j}$-component of the corresponding term in the new equation can be made equal to zero.

After performing a normalisation with parameters $(C=\mathrm{id}, a=0, r=$ $\left.\left(r_{1}, r_{2}\right)\right)$ with suitable $r_{j}$ the condition that involves the $\mathcal{Q}^{j}$ will also be satisfied.

Now we turn to the proof of the uniqueness statement. By inspection one easily verifies that any linear mapping of the form (2) preserves the s.n.f. Now, let $\Phi$ be a local coordinate change such that the new equation 
also has s.n.f. Then $\Phi$ is a renormalisation and its linear part is equal to some $L_{C}$. The mapping $L_{C}^{-1} \circ \Phi$ is also a coordinate change that takes $M$ to s.n.f. and its linear part is the identity. Such renormalisations act on the first non-quadratic terms like quadratic automorphisms and, therefore, would produce non-normal terms if one of the $a_{j}, r_{j}$ were different from 0 . This shows that $a_{j}=r_{j}=0$. But, according to Loboda's theorem, then $L_{C}^{-1} \circ \Phi=$ id, i.e., $\Phi=L_{C}$.

\section{References}

[CM74] S. S. Chern and J. Moser, Real hypersurfaces in complex manifolds, Acta Math. 133 (1974), 219-271.

[Lob88] A. V. Loboda, Generic real analytic manifolds of codimension 2 in $\mathbb{C}^{4}$ and their biholomorphic mappings, Izv. Akad. Nauk SSSR Ser. Mat. 52 (1988), 970-990 (in Russian); English transl.: Math. USSR-Izv. 33 (1989), 295-315.

[Lob90] - Linearizability of holomorphic mappings of generating manifolds of codimension 2 in $\mathbb{C}^{4}$, Izv. Akad. Nauk SSSR Ser. Mat. 54 (1990), 632-644 (in Russian); English transl.: Math. USSR-Izv. 36 (1991), 655-667.

[Poi07] H. Poincaré, Les fonctions analytiques de deux variables et la représentation conforme, Rend. Circ. Mat. Palermo 23 (1907), 185-220.

[Sch95] G. Schmalz, CR-Mannigfaltigkeiten höherer Kodimension und ihre Automorphismen, Habilitation thesis, Rheinische Friedrich-Wilhelms-Universität Bonn, 1995, to appear in Math. Nachr.

[Web78] S. M. Webster, On the Moser normal form at a non-umbilic point, Math. Ann. 233 (1978), 97-102.

University of Adelaide

Department of Pure Mathematics

Adelaide, South Australia 5005

Australia

E-mail: vezhov@maths.adelaide.edu.au
Mathematisches Institut der Universität Bonn Beringstraße 1 D-53115 Bonn, Germany E-mail:schmalz@uni-bonn.de 